\title{
Can Non-neurological Intrinsic Factors Affect Outcome of TURP in Patients with BPH?
}

\section{Ganesh Sonawane ${ }^{1}$, Biju S Pillai ${ }^{2}$ and H Krishna Moorthy ${ }^{2 *}$}

${ }^{1}$ Senior Resident, Division of Urology, Lourdes Institute of Nephro-Urology, Lourdes

Hospital, Kochi, India

${ }^{2}$ Consultant Urologists, Division of Urology, Lourdes Institute of Nephro-Urology,

Lourdes Hospital, Kochi, India

*Corresponding Author: H Krishna Moorthy, Consultant Urologists, Division of Urology, Lourdes Institute of Nephro-Urology, Lourdes Hospital, Kochi, India.
Received: July 25, 2021

Published: August 30, 2021

(C) All rights are reserved by $\mathbf{H}$ Krishna

Moorthy., et al.

\section{Abstract}

Introduction: Transurethral resection of prostate (TURP) is the mainstay of surgery for Benign Prostatic Hyperplasia (BPH). However it is estimated that upto $20 \%$ of patients have unfavourable results after TURP, suggesting certain other factors affecting outcome of TURP. Neurological changes occurring in the urinary bladder in patients with long standing BPH have been widely postulated as the reason for this poor outcome in some patients. This study was undertaken to assess the role of various intrinsic factors other than neurological causes, influencing outcome of TURP for BPH.

Materials and Methods: This was a prospective study on 120 cases of BPH qualifying inclusion criteria with absolute indications for TURP. Detailed physical, sonological and biochemical evaluation were done pre and post ( 1 and 3 months) TURP. The results of surgery were compared in various groups to evaluate the role of intrinsic factors like age of patients, prostate size, UTI, catheterization status, grades of diverticula, presence of diverticula and post void residue (PVR) in the outcome of TURP.

Results: There was significant difference in symptom score and flow patterns in pre and post operative patients classified into different groups according to the age, presence of UTI and PVR volume. The post operative flow rates were significantly lower in grade III trabeculation patients compared to other groups. The post operative symptom score was significantly lower and flow rates were higher in the group without diverticula.

Conclusions: Elderly patients (>65years) and patients without UTI before TURP had better functional outcome after TURP. Larger glands (>45grams) had favourable outcome, while poorer outcome was found in patients with higher grades of bladder trabeculations. However, there was no difference in outcome between catheterised and non-catheterised patients. Therefore there is significant role of non-neurological factors in the outcome of TURP for BPH.

Keywords: Benign Prostatic Hyperplasia; Trans Urethral Resection of Prostate; Urodynamics; International Prostate Symptom Score

\section{Introduction}

Benign Prostate Hyperplasia (BPH) is a common disease in adult men associated with bothersome lower urinary tract symptoms (LUTS) and its incidence is age related. Prevalence of BPH is approximately $25 \%$ in men aged 40 to 49 years, 50\% in men aged 50 to 59 years and $80 \%$ in men aged 70 to 79 years [1]. Although $\mathrm{BPH}$ is not caused by androgens, the postulated theory is that the presence of androgens is needed for the pathogenesis. Other etio-

Citation: H Krishna Moorthy., et al. "Can Non-neurological Intrinsic Factors Affect Outcome of TURP in Patients with BPH?". Acta Scientific Neurology 4.10 (2021): 77-85. 
pathological causes postulated for BPH include age-related tissue changes, metabolic syndrome, hormonal alterations, inflammation etc. [2].

The most common presenting complication of BPH that requires hospitalization is acute urinary retention (AUR), which is an important health issue. Many complications of BPH like recurrent urinary tract infections (UTIs), formation of bladder calculi, hematuria, and damage to bladder wall and kidneys develop when there is chronic urinary obstruction due to BPH. Although BPH is generally not a life threatening condition, it can have a marked effect on patient's Quality Of Life (QOL) [3].

Minimally invasive procedures including Transurethral Resection of Prostate (TURP) and Transurethral Laser Enucleation of Prostate (TULEP) have become mainstay of operative management of BPH. Open surgery is currently being reserved for larger glands or when BPH is associated with other complications like a large bladder stone. In spite of all developments in surgical crafts, TURP is still considered as gold standard for the management of BPH [5]. It is estimated that TURP is the second most common surgery performed next to cataract surgery in male patients older than 50 years of age.

The outcome of TURP can be assessed by changes in various subjective parameters like Boyarsky score, Madsen Iversen score, American Urological Association (AUA) symptom index, Danish prostatic symptom score, International Prostatic Symptom Score (IPSS Score) and objective parameters like Urodynamic and uroflowmetry parameters including Maximum Flow Rate (Qmax) and Average Flow Rate (Qav). Though regarded as gold standard, it is discerning to note that upto $20 \%$ of patients have unfavourable results after TURP [4]. This points to the fact that various other intrinsic factors also influence the results of TURP for troublesome $\mathrm{BPH}$. Various neurological changes in the form of hyper or hypoactivity has been established to develop in the urinary bladder in patients with urinary obstruction due to long standing BPH. Many scientists have suggested undertaking urodynamic studies (UDS) before embarking on TURP in patients with BPH of long duration symptoms [5-7]. However a recent study showed that routine use of UDS in men undergoing investigation for LUTS was not useful and it could only be advised for selective utilisation in individual cases to assess the long-term outcomes of diagnosis and therapy [8]. Moreover the urodynamic changes mostly revert after a few months of surgery in these patients.
Therefore there is an urgent need to study the factors other than neurological changes in urinary bladder in patients with urinary obstruction, before we undertake TURP for treatment of BPH and reassure the patient regarding the results of procedure.

This study was undertaken to assess various non-neurological pre operative and intra operative intrinsic factors which would influence the post operative outcome in patients undergoing TURP for $\mathrm{BPH}$.

\section{Materials and Methods}

This was a prospective study on patients with BPH having absolute indication for TURP, aged between 50 yrs to 80 yrs and fit for surgery. Patients with carcinoma prostate, coexistent urothelial malignancy, neurological disorders and those on medicines which affect bladder contractility, long standing uncontrolled diabetes mellitus (>8-10 years), other coexistent diseases like chronic kidney disease, upper tract pathologies, stricture urethra etc, past history of surgeries in prostate and patients undergoing incomplete resection/staged TURP were excluded from the study. Patients with symptoms of BPH of more than 1 year were also excluded, to avoid the neurological factors in bladder due to long standing $\mathrm{BPH}$ as a confounding factor in the results of TURP. After obtaining Institutional Ethical Committee clearance, a total of 120 patients fulfilling inclusion criteria were enrolled in this study. The diagnosis of BPH was confirmed by clinical and radiological evaluation including digital rectal examination (DRE). Patient age and associated comorbid conditions were recorded. Based on age, the patients were classified into two groups - those below 65 yrs and those above 65 years. In patients who presented with AUR, urinary symptoms prior to developing AUR were recorded. Post operatively, IPSS score assessment was done in all patients at 1 month and 3 months after TURP.

Patients were classified into four groups depending upon size of prostate on DRE (Grade I,II,III,IV) [4]. Routine blood investigations like complete haemogram, serum creatinine and random blood sugar were done before the procedure. Routine urine analysis and urine culture were done in all patients to rule out urinary tract infection. Urine culture was done by collecting the mid stream voided urine in patients without AUR. For patients with AUR, urine sample was collected from urethral catheter. If culture was found to be positive, appropriate antibiotic was given for one week before the procedure and the patients were taken up for surgery. Patients 
were classified into two groups - those who had UTI prior to surgery and those without UTI.

Serum PSA was measured in all patients as a marker of carcinoma prostate. In patients with catheter, serum PSA was done after appropriate antibiotic therapy for one month. If the PSA was above $4 \mathrm{ng} / \mathrm{ml}$, or if the percentage of free PSA was less than $25 \%$ along with an abnormal DRE finding, prostate biopsy was done to rule out malignancy. Once the patient was found to have biopsy proved malignancy, he was excluded from the study.

Preoperatively Uroflowmetry was done in all patients (except patients who presented with AUR) to assess the flow pattern including Maximum flow rate (Qmax) and Average flow rate (Qav). Patients who were suspicious to have stricture urethra underwent ascending urothrogram and when confirmed, were excluded from the study. If there was any suspicion of neurogenic bladder, urodynamic evaluation was done. Patients with neurogenic bladder were excluded from the study. Uroflow assessment was done in all patients at 1 month and 3 months after TURP.

Transabdominal Ultra sonogram (USG) was done preoperatively to measure the prostate size in all patients and to assess the post void residue (PVR) in patients who were not on catheter. Based on USG, the patients were grouped into those with $>45$ gm prostate and those with less than $45 \mathrm{gm}$ prostate volume and those with PVR $>60 \mathrm{ml}$ and less than $60 \mathrm{ml}$. USG was performed post operatively to measure PVR at 1 month and 3 months post operatively.

All patients fulfilling inclusion criteria underwent standard, complete monopolar TURP under spinal anesthesia performed by a single senior Urologist having $>10$ yrs of expertise. Catheter was removed in all patients on 4 th post operative day. Based on grades of trabeculations assessed during cystoscopy, the patients were classified into four groups (grade 0, I, II and III - none, mild, moderate and severe trabeculations).

Un paired student $t$ test was used to find out the significance of difference between proportion and percentage of the results obtained. Repeated measure ANOVA was applied to calculate significance of pre operative intrinsic factors on postoperative outcome.

\section{Results}

Out of the 120 patients, 50 belonged to the age group less than
65 years and the rest were above 65 yrs. The mean age of the patients was found to be $67.2 \pm 7.14$ years.

On DRE, 20 patients had grade I prostatomegaly 72 patients had grade II and 28 had grade III prostatomegaly. Based on USG, the mean prostate size was found to be $55.63 \pm 14.03$ grams (range: 28-86 grams).

Based on intra operative cystoscopic findings of trabeculations grading, 50 patients had grade I, 30 patients had grade II, 12 patients had grade III trabeculations and 28 patients had no trabeculations. 38 patients had coexistent diverticuli.

90 patients had urinary tract infection prior to surgery and were on antibiotics for a minimum of 1 week prior to surgery.

Significant decrease in IPSS was noted post operatively in all patients ( $\mathrm{p}$ < 0.05), while Qmax and Qav increased significantly on post operative assessment at 1 month and 3 months compared to baseline in non-catheterised patients $(\mathrm{p}<0.05)$. Table 1 depicts the change in IPSS in pre and post operative settings.

\begin{tabular}{|l|c|c|c|}
\hline & Mean IPSS & Mean Qmax & Mean Qav \\
\hline Pre operative & $26.65 \pm 3.56$ & $8.68 \pm 1.88$ & $3.34 \pm 0.52$ \\
\hline $\begin{array}{l}\text { Post operative }(1 \\
\text { month) (ml/s) }\end{array}$ & $12.43 \pm 2.66$ & $19.05 \pm 2.18$ & $7.13 \pm 1.3$ \\
\hline $\begin{array}{l}\text { Post operative (3 } \\
\text { months) (ml/s) }\end{array}$ & $12.70 \pm 3.74$ & $18.32 \pm 3.07$ & $16.76 \pm 1.40$ \\
\hline p value & $<0.01$ & $<0.01$ & $<0.01$ \\
\hline
\end{tabular}

Table 1: Change in IPSS ( $\mathrm{n}=120)$.

IPSS: International symptom score, Qmax: Maximum urinary flow rate, Qav: Average urinary flow rate.

$\mathrm{p}$ value calculate by Repeated measures ANOVA.

Qmax and Qav comparison done only for preoperatively non-catherized patients $(n=48)$.

There was a significant reduction in PVR volume in 48 non catheterised patients at 1 month and 3 months post operatively in comparison to the pre surgery mean values. However no statistical difference was found between the values measured at 1 month and 3 months in post-hoc analysis ( $\mathrm{p}>0.05$ ) (Table 2).

Citation: H Krishna Moorthy., et al. "Can Non-neurological Intrinsic Factors Affect Outcome of TURP in Patients with BPH?". Acta Scientific Neurology 4.10 (2021): 77-85. 


\begin{tabular}{|l|c|}
\hline & $\begin{array}{c}\text { Mean Post-void residual urine } \\
\text { volume (ml) }\end{array}$ \\
\hline Pre operative & $92.37 \pm 52.84$ \\
\hline Post operative (1 month) & $28.83 \pm 22.1$ \\
\hline Post operative (3months) & $34.85 \pm 26.9$ \\
\hline p value & $<0.01$ \\
\hline
\end{tabular}

Table 2: Changes in Post Void Residual volume $(n=48)$.

$\mathrm{p}<0.05$ considered significant by Repeated measures ANOVA Test.

Results of analysis of functional outcomes correlated with intrinsic factors

\section{Age of patients}

Based on functional outcome parameters, post operative IPSS was found to be significantly higher (poorer outcome) in the patient group $<65$ years at 3 months post operative ( $p<0.05$ ). However, the post operative Qmax and Qav were significantly higher (favourable outcome) in the age group $>65$ yrs. Table 3 shows the details.

\begin{tabular}{|l|c|c|c|}
\hline & $\begin{array}{c}\text { Age of patients } \\
\text { (<65 years) } \\
\text { [n= 50] }\end{array}$ & $\begin{array}{c}\text { Age of patients } \\
\text { (>65years) } \\
\text { [n= 70] }\end{array}$ & $\begin{array}{c}\text { p } \\
\text { value }\end{array}$ \\
\hline Pre operative IPSS & $26.32 \pm 4.5$ & $26.89 \pm 2.74$ & 0.53 \\
\hline $\begin{array}{l}\text { Post operative } \\
\text { IPSS (1 month) }\end{array}$ & $12.52 \pm 2.86$ & $12.37 \pm 2.56$ & 0.61 \\
\hline $\begin{array}{l}\text { Post operative } \\
\text { IPSS (3 months) }\end{array}$ & $13.76 \pm 4.93$ & $11.94 \pm 2.4$ & 0.03 \\
\hline $\begin{array}{l}\text { Pre operative } \\
\text { Qmax (ml/sec) }\end{array}$ & $8.88 \pm 1.83$ & $8.54 \pm 1.93$ & 0.46 \\
\hline $\begin{array}{l}\text { Post operative } \\
\text { Qmax (1 month) } \\
\text { (ml/sec) }\end{array}$ & $19.04 \pm 2.15$ & $19.06 \pm 2.24$ & 0.81 \\
\hline $\begin{array}{l}\text { Post operative } \\
\text { Qmax (3 months) } \\
\text { (ml/sec) }\end{array}$ & $17.68 \pm 3.91$ & $19.77 \pm 2.25$ & 0.02 \\
\hline $\begin{array}{l}\text { Pre operative Qav } \\
\text { (ml/sec) }\end{array}$ & $3.33 \pm 0.53$ & $3.34 \pm 0.52$ & 0.91 \\
\hline $\begin{array}{l}\text { Post operative Qav } \\
\text { (1 month) (ml/ } \\
\text { sec) }\end{array}$ & $7.3 \pm 1.48$ & $7.11 \pm 1.18$ & 0.45 \\
\hline $\begin{array}{l}\text { Post operative Qav } \\
\text { (3 months) (ml/ } \\
\text { sec) }\end{array}$ & $6.56 \pm 1.64$ & $6.93 \pm 1.23$ & 0.04 \\
\hline
\end{tabular}

Table 3: Effect of age on functional outcomes of surgery.

IPSS: International prostate symptom score, Qmax: maximum urinary flow rate, Qav: average urinary flow rate.

$\mathrm{p}<0.05$ considered significant by unpaired t test.

Pre operative Qmax and Qav calculated only for non-catheterized patients.

\section{Presence of UTI}

Post operative IPSS was found to be significantly higher (poorer outcome) in the UTI-positive group at 3 months post operative (p $<0.05$ ). The post operative Qmax and Qav were also significantly lower (poorer outcome) in the UTI-positive group (Table 4).

\begin{tabular}{|l|c|c|c|}
\hline & $\begin{array}{c}\text { Absence of } \\
\text { UTI } \\
\text { (n= 30) }\end{array}$ & $\begin{array}{c}\text { Presence of } \\
\text { UTI } \\
\text { (n=90) }\end{array}$ & p value \\
\hline Pre operative IPSS & $24.63 \pm 3.9$ & $26.39 \pm 3.12$ & 0.09 \\
\hline $\begin{array}{l}\text { Post operative IPSS } \\
\text { (1 month) }\end{array}$ & $11.19 \pm 2.4$ & $12.89 \pm 2.63$ & 0.04 \\
\hline $\begin{array}{l}\text { Post operative IPSS } \\
\text { (3 months) }\end{array}$ & $11.44 \pm 2.76$ & $13.16 \pm 3.97$ & 0.01 \\
\hline $\begin{array}{l}\text { Pre operative Qmax } \\
\text { (ml/sec) }\end{array}$ & $9.31 \pm 1.92$ & $8.45 \pm 1.84$ & 0.16 \\
\hline $\begin{array}{l}\text { Post operative Qmax } \\
\text { (1 month) (ml/sec) }\end{array}$ & $18.81 \pm 2.07$ & $19.14 \pm 2.24$ & 0.23 \\
\hline $\begin{array}{l}\text { Post operative Qmax } \\
\text { (3 months) (ml/sec) }\end{array}$ & $18.63 \pm 2.8$ & $18.1 \pm 3.18$ & 0.04 \\
\hline $\begin{array}{l}\text { Pre operative Qav } \\
\text { (ml/sec) }\end{array}$ & $3.36 \pm 0.53$ & $3.33 \pm 0.52$ & 0.89 \\
\hline $\begin{array}{l}\text { Post operative Qav (1 } \\
\text { month) (ml/sec) }\end{array}$ & $7.45 \pm 1.48$ & $7.01 \pm 1.28$ & 0.04 \\
\hline $\begin{array}{l}\text { Post operative Qav (3 } \\
\text { months) (ml/sec) }\end{array}$ & $7.39 \pm 1.32$ & $6.53 \pm 1.37$ & 0.01 \\
\hline
\end{tabular}

Table 4: Effect of UTI on functional outcomes of surgery.

IPSS: International prostate symptom score, Qmax: maximum urinary flow rate, Qav: average urinary flow rate.

$\mathrm{P}<0.05$ considered significant by unpaired $\mathrm{t}$ test.

Pre- op Qmax and Qav calculated only for non-catheterized patients.

Status of indwelling catheter

No statistical difference was found in various parameters measured between patients with catheter and without catheter ( $\mathrm{p}>0.05)$ as shown in table 5.

\section{Preoperative post-void residue}

Post operative IPSS was found to be significantly higher (poorer outcome) in the group where the PVR was $>60 \mathrm{ml}$ at both 1 month and 3 months post operative. The post operative Qmax and Qav were comparable in both the study groups $(p>0.05)$ (Table 6).

Sonologically measured size of prostate

Post operative IPSS was found to be comparable in both the study groups at every point of measurement of parameter. The post operative Qmax and Qav were found to be significantly higher (fa- 
vourable outcome) in the $>45$ grams prostate size group $(\mathrm{p}<0.05)$

(Table 7).

\begin{tabular}{|l|c|c|c|}
\hline & $\begin{array}{c}\text { No catheter } \\
\text { (n= 48) }\end{array}$ & $\begin{array}{c}\text { Catheter } \\
\text { Present } \\
\text { (n= 72) }\end{array}$ & $\begin{array}{c}\mathbf{p} \\
\text { value }\end{array}$ \\
\hline Pre operative IPSS & $26.08 \pm 3.99$ & $27.03 \pm 3.24$ & 0.17 \\
\hline $\begin{array}{l}\text { Post operative IPSS (1 } \\
\text { month) }\end{array}$ & $11.88 \pm 2.38$ & $12.41 \pm 2.81$ & 0.23 \\
\hline $\begin{array}{l}\text { Post operative IPSS (3 } \\
\text { months) }\end{array}$ & $12.33 \pm 3.58$ & $12.84 \pm 3.88$ & 0.32 \\
\hline $\begin{array}{l}\text { Post operative Qmax (1 } \\
\text { month) (ml/sec) }\end{array}$ & $19.17 \pm 2.65$ & $18.97 \pm 1.84$ & 0.52 \\
\hline $\begin{array}{l}\text { Post operative Qmax (3 } \\
\text { months) (ml/sec) }\end{array}$ & $18.79 \pm 3.24$ & $18.31 \pm 2.95$ & 0.36 \\
\hline $\begin{array}{l}\text { Post operative Qav (1 } \\
\text { month) (ml/sec) }\end{array}$ & $7.19 \pm 1.37$ & $7.09 \pm 1.29$ & 0.71 \\
\hline $\begin{array}{l}\text { Post operative Qav (3 } \\
\text { months) (ml/sec) }\end{array}$ & $6.93 \pm 1.46$ & $6.75 \pm 1.37$ & 0.26 \\
\hline
\end{tabular}

Table 5: Effect of Catheterization on functional outcomes of surgery.

IPSS: International prostate symptom score, Qmax: maximum urinary flow rate, Qav: average urinary flow rate.

$\mathrm{P}>0.05$ considered not significant by unpaired $t$ test.

\begin{tabular}{|l|c|c|c|}
\hline & $\begin{array}{c}\text { PVR }<60 \text { ml } \\
(\mathbf{n}=\mathbf{1 8})\end{array}$ & $\begin{array}{c}\text { PVR }>\mathbf{6 0} \text { ml } \\
\text { (n= 30) }\end{array}$ & $\begin{array}{c}\mathbf{p} \\
\text { value }\end{array}$ \\
\hline Pre operative IPSS & $25.61 \pm 3.55$ & $27.1 \pm 3.45$ & 0.13 \\
\hline $\begin{array}{l}\text { Post operative IPSS (1 } \\
\text { month) }\end{array}$ & $11.43 \pm 2.71$ & $13.05 \pm 2.47$ & 0.03 \\
\hline $\begin{array}{l}\text { Post operative IPSS (3 } \\
\text { months) }\end{array}$ & $12.04 \pm 3.9$ & $13.21 \pm 3.63$ & 0.02 \\
\hline $\begin{array}{l}\text { Post operative Qmax } \\
\text { (1 month) (ml/sec) }\end{array}$ & $18.96 \pm 2.21$ & $19.01 \pm 2.21$ & 0.79 \\
\hline $\begin{array}{l}\text { Post operative Qmax } \\
\text { (3 months) (ml/sec) }\end{array}$ & $18.48 \pm 2.76$ & $18.32 \pm 2.98$ & 0.48 \\
\hline $\begin{array}{l}\text { Post operative Qav (1 } \\
\text { month) (ml/sec) }\end{array}$ & $7.27 \pm 1.17$ & $7.14 \pm 1.41$ & 0.53 \\
\hline $\begin{array}{l}\text { Post operative Qav (3 } \\
\text { months) (ml/sec) }\end{array}$ & $6.88 \pm 1.16$ & $6.79 \pm 1.54$ & 0.77 \\
\hline
\end{tabular}

Table 6: Pre operative post-void residue (PVR) on functional outcomes of surgery $(n=48)$.

IPSS: International prostate symptom score, Qmax: maximum urinary flow rate, Qav: average urinary flow rate. $\mathrm{p}<0.05$ considered significant by unpaired $t$ test.

\begin{tabular}{|l|c|c|c|}
\hline & $\begin{array}{c}\text { Prostate Size } \\
\mathbf{> 4 5} \\
\text { grams (n = 32) }\end{array}$ & $\begin{array}{c}\text { Prostate Size } \\
\mathbf{4 4 5}\end{array}$ & $\begin{array}{c}\text { prams (n = 88) } \\
\text { value }\end{array}$ \\
\hline Pre operative IPSS & $25.13 \pm 3.88$ & $26.2 \pm 3.3$ & 0.11 \\
\hline $\begin{array}{l}\text { Post operative IPSS } \\
\text { (1 month) }\end{array}$ & $11.69 \pm 2.52$ & $12.17 \pm 2.69$ & 0.14 \\
\hline $\begin{array}{l}\text { Post operative IPSS } \\
\text { (3 months) }\end{array}$ & $11.94 \pm 3.01$ & $12.28 \pm 3.97$ & 0.38 \\
\hline $\begin{array}{l}\text { Pre operative } \\
\text { Qmax (ml/sec) }\end{array}$ & $9.63 \pm 1.75$ & $8.94 \pm 1.83$ & 0.56 \\
\hline $\begin{array}{l}\text { Post operative } \\
\text { Qmax (1 month) } \\
\text { (ml/sec) }\end{array}$ & $19.31 \pm 1.78$ & $18.95 \pm 2.32$ & 0.51 \\
\hline $\begin{array}{l}\text { Post operative } \\
\text { Qmax (3 months) } \\
\text { (ml/sec) }\end{array}$ & $19.25 \pm 2.57$ & $17.89 \pm 3.19$ & 0.03 \\
\hline $\begin{array}{l}\text { Pre operative Qav } \\
\text { (ml/sec) }\end{array}$ & $3.48 \pm 0.43$ & $3.38 \pm 0.54$ & 0.72 \\
\hline $\begin{array}{l}\text { Post operative Qav } \\
\text { (1 month) (ml/sec) }\end{array}$ & $7.64 \pm 1.17$ & $6.94 \pm 1.18$ & 0.41 \\
\hline $\begin{array}{l}\text { Post operative Qav } \\
\text { (3 months) (ml/ } \\
\text { sec) }\end{array}$ & $7.53 \pm 1.24$ & $6.88 \pm 1.23$ & 0.04 \\
\hline
\end{tabular}

Table 7: Effect of Prostate size based on USG on functional outcomes of surgery.

IPSS: International prostate symptom score, Qmax: maximum urinary flow rate, Qav: average urinary flow rate. $\mathrm{p}<0.05$ considered significant by unpaired $\mathrm{t}$ test.

Pre- op Qmax and Qav calculated only for non-catheterized patients.

Prostate size measured by DRE

Patients with Grade III size on DRE were found to have significantly higher IPSS (poorer outcome) on follow-up ( $p<0.05)$. Though the post operative Qmax were comparable in all three groups, the Qav was significantly lower in grade III patients on follow-up (Table 8).

\section{Grades of trabeculations}

The post operative Qmax and Qav were found to be significantly lower (poorer outcome) in the grade III patients as compared to other groups $(\mathrm{p}<0.05)$ (Table 9).

\section{Presence of diverticula}

Post operative IPSS was found to be significantly lower (favourable outcome) in the group without diverticula $(\mathrm{p}<0.05)$. The post operative Qmax and the Qav were found to be significantly higher in the group without diverticula $(\mathrm{p}<0.05)$. The details are given in table 10. 


\begin{tabular}{|l|c|c|c|c|}
\hline & Grade I (n= 20) & Grade II (n = 72) & Grade III (n= 28) & p value \\
\hline Pre operative IPSS & $24.8 \pm 4.37$ & $26.44 \pm 3.32$ & $28.5 \pm 2.85$ & 0.01 \\
\hline Post operative IPSS (1 month) & $11.9 \pm 2.47$ & $12.03 \pm 2.49$ & $13.86 \pm 2.91$ & 0.02 \\
\hline Post operative IPSS (3 months) & $12.1 \pm 2.91$ & $12.28 \pm 3.69$ & $14.14 \pm 4.26$ & 0.01 \\
\hline Pre operative Qmax (ml/sec) & $9.4 \pm 1.84$ & $8.89 \pm 1.79$ & $8.64 \pm 1.86$ & 0.09 \\
\hline $\begin{array}{l}\text { Post operative Qmax (1 } \\
\text { month) (ml/sec) }\end{array}$ & $18.9 \pm 1.91$ & $19.03 \pm 1.97$ & $19.07 \pm 2.95$ & 0.67 \\
\hline $\begin{array}{l}\text { Post operative Qmax (3 } \\
\text { months) (ml/sec) }\end{array}$ & $18.4 \pm 2.22$ & $18.23 \pm 3.29$ & $18.14 \pm 3.18$ & 0.56 \\
\hline Pre operative Qav (ml/sec) & $3.53 \pm 0.42$ & $3.4 \pm 0.49$ & $3.04 \pm 0.55$ & 0.12 \\
\hline Post operative Qav (1 month) (ml/sec) & $7.46 \pm 0.98$ & $7.35 \pm 1.38$ & $6.33 \pm 1.03$ & 0.03 \\
\hline Post operative Qav (3 months) (ml/sec) & $7.31 \pm 0.92$ & $6.97 \pm 1.48$ & $5.84 \pm 1.08$ & 0.01 \\
\hline
\end{tabular}

Table 8: Effect of prostate size based on DRE on functional outcomes of surgery.

IPSS: International prostate symptom score, Qmax: maximum urinary flow rate, Qav: average urinary flow rate.

$\mathrm{p}<0.05$ considered significant by unpaired $\mathrm{t}$ test.

Pre operative Qmax and Qav calculated only for non-catheterized patients.

\begin{tabular}{|c|c|c|c|c|c|}
\hline & $\begin{array}{l}\text { Grade } 0 \\
(n=28)\end{array}$ & $\begin{array}{l}\text { Grade I } \\
(\mathrm{n}=\mathbf{5 0})\end{array}$ & $\begin{array}{l}\text { Grade II } \\
(\mathrm{n}=\mathbf{3 0})\end{array}$ & $\begin{array}{l}\text { Grade III } \\
(\mathrm{n}=12)\end{array}$ & p value \\
\hline Pre operative IPSS & $26.64 \pm 3.23$ & $25.44 \pm 3.71$ & $27.6 \pm 2.97$ & $29.33 \pm 3.56$ & 0.01 \\
\hline Post operative IPSS (1 month) & $12.24 \pm 1.7$ & $12.32 \pm 2.87$ & $12.93 \pm 2.4$ & $12.33 \pm 4.41$ & 0.21 \\
\hline Post operative IPSS (3 months) & $12.1 \pm 1.81$ & $13.12 \pm 4.61$ & $12.7 \pm 3.81$ & $12.34 \pm 3.44$ & 0.09 \\
\hline Pre operative $\mathrm{Qmax}(\mathrm{ml} / \mathrm{sec})$ & $9.21 \pm 1.97$ & $9.08 \pm 1.91$ & $7.8 \pm 0.94$ & $9 \pm 2.61$ & 0.11 \\
\hline $\begin{array}{l}\text { Post operative Qmax (1 month) } \\
\text { (ml/sec) }\end{array}$ & $19.07 \pm 1.64$ & $19.21 \pm 2.1$ & $19.33 \pm 2.55$ & $17.67 \pm 2.66$ & 0.13 \\
\hline $\begin{array}{l}\text { Post operative Qmax (3 months) } \\
\text { (ml/sec) }\end{array}$ & $19.08 \pm 2.23$ & $18.24 \pm 2.99$ & $18.47 \pm 3.89$ & $16.5 \pm 2.66$ & 0.02 \\
\hline Pre operative Qav (ml/sec) & $3.45 \pm 0.47$ & $3.36 \pm 0.52$ & $3.35 \pm 0.43$ & $2.95 \pm 0.76$ & 0.14 \\
\hline $\begin{array}{l}\text { Post operative Qav (1 month) } \\
\text { (ml/sec) }\end{array}$ & $7.13 \pm 1.53$ & $7.27 \pm 1.14$ & $7.1 \pm 1.21$ & $6.27 \pm 1.74$ & 0.03 \\
\hline $\begin{array}{l}\text { Post operative Qav (3 months) } \\
\text { (ml/sec) }\end{array}$ & $7.22 \pm 1.65$ & $6.85 \pm 1.27$ & $6.57 \pm 1.35$ & $6.35 \pm 1.57$ & 0.01 \\
\hline
\end{tabular}

Table 9: Effect of grades of trabeculation on functional outcomes of surgery.

IPSS: International prostate symptom score, Qmax: maximum urinary flow rate, Qav: average urinary flow rate.

$$
\mathrm{p}<0.05 \text { considered significant by unpaired t test. }
$$




\begin{tabular}{|l|c|c|c|}
\hline & $\begin{array}{c}\text { Diverticula } \\
\text { present } \\
\text { (n= 38) }\end{array}$ & $\begin{array}{c}\text { Diverticula } \\
\text { absent } \\
\text { (n= 82) }\end{array}$ & $\begin{array}{c}\mathbf{p} \\
\text { value }\end{array}$ \\
\hline Pre operative IPSS & $28.53 \pm 3.17$ & $27.78 \pm 3.42$ & 0.41 \\
\hline $\begin{array}{l}\text { Post operative IPSS } \\
\text { (1 month) }\end{array}$ & $13.26 \pm 3.46$ & $12.05 \pm 2.14$ & 0.03 \\
\hline $\begin{array}{l}\text { Post operative IPSS } \\
\text { (3 months) }\end{array}$ & $13.74 \pm 4.85$ & $12.22 \pm 3.05$ & 0.04 \\
\hline $\begin{array}{l}\text { Pre operative Qmax } \\
\text { (ml/sec) }\end{array}$ & $7.89 \pm 1.82$ & $8.05 \pm 1.82$ & 0.47 \\
\hline $\begin{array}{l}\text { Post operative Qmax } \\
\text { (1 month) (ml/sec) }\end{array}$ & $18.79 \pm 2.32$ & $19.17 \pm 2.13$ & 0.08 \\
\hline $\begin{array}{l}\text { Post operative Qmax } \\
\text { (3 months) (ml/sec) }\end{array}$ & $17.11 \pm 3.62$ & $18.88 \pm 2.64$ & 0.02 \\
\hline $\begin{array}{l}\text { Pre operative Qav } \\
\text { (ml/sec) }\end{array}$ & $3.22 \pm 0.58$ & $3.39 \pm 0.49$ & 0.39 \\
\hline $\begin{array}{l}\text { Post operative Qav } \\
\text { (1 month) (ml/sec) }\end{array}$ & $6.87 \pm 1.40$ & $7.25 \pm 1.27$ & 0.19 \\
\hline $\begin{array}{l}\text { Post operative Qav } \\
\text { (3 months) (ml/sec) }\end{array}$ & $6.25 \pm 1.49$ & $7 \pm 1.31$ & 0.01 \\
\hline
\end{tabular}

Table 10: Effect of diverticula on functional outcomes of surgery.

IPSS: International prostate symptom score, Qmax: maximum urinary flow rate, Qav: average urinary flow rate.

$\mathrm{p}<0.05$ considered significant by unpaired t test.

Pre op Qmax and Qav calculated only for non-catheterized patients.

\section{Discussion}

TURP has evolved as the gold standard in surgical treatment of BPH due to its excellent, well-documented, and long term efficacy. However it has been reported that $20 \%$ of the patients had unfavorable results after TURP $[4,9,10]$. It is important to identify preoperative and intra-operative factors that may impact the degree of success of TURP, so that the influence of these factors could be considered before deciding the type of treatment modality adopted in such patients. Neurological changes in the urinary bladder in the form of detrusor over or underactivity have been described as the main cause for these poor results and therefore the role of preoperative urodynamic tests had been advocated to identify these changes prior to TURP. However many researchers found out that the poor results of TURP were also observed in patients with short term urinary obstruction also questioning the impact of neurologi- cal element as a confounding factor in the results. Literature search revealed a dearth of studies which have tried to analyze the impact of various non neurological intrinsic factors on outcome of TURP.

Significant decrease in IPSS was noted post operatively $(\mathrm{p}<$ 0.05 ) in all patients, while Qmax and Qav increased significantly on post operative assessment at 1 month and 3 months compared to baseline in patients without catheter. In the study by Milanos., et al. [11] similar results were noted. The IPSS decreased significantly at 6-month evaluation in the Milanos., et al. study, while the Qmax increased significantly as well. In the study by Hakenberg., et al. [12], the mean pre operative IPSS was $19.68 \pm 0.83$ which decreased at 3-month postop follow up to $9.75 \pm 0.96$. The pre operative Qmax in this study was found to be $10.3 \mathrm{ml} / \mathrm{s}$ which increased to 17.36 $\mathrm{ml} / \mathrm{s}$ at 3 month-follow-up. In the study by Hossain., et al. [13], the mean IPSS of preoperative, 1st month and 3rd month were $25.18 \pm$ $1.45,15.00 \pm 1.07$ and $8.14 \pm 0.76$ respectively. The mean ( \pm SD) of the $\mathrm{Qmax}$ was $9.70 \pm 1.20 \mathrm{ml} / \mathrm{sec}, 15.78 \pm 1.42 \mathrm{ml} / \mathrm{sec}$, and $18.78 \pm$ $1.33 \mathrm{ml} / \mathrm{sec}$ in pre operative, $1 \mathrm{st}$ month, and $3 \mathrm{rd}$ month respectively. On literature search it was noted that most studies calculated either Qmax or Qav, while we calculated both the values which is actually a more holistic view of the urinary flow rate post surgery.

Though not many studies have evaluated the relation of factors various non-neurological intrinsic factors with post TURP outcomes, few studies which have had similar findings as ours. In our study, elderly patients $>65$ years had better results although various studies have shown that increased age of patients undergoing TURP may have poorer outcomes. Possible explanation given was prevalence of instrinsic detrusor underactivity rising with age, juxtaposed by a decrease of bladder outflow obstruction. Although detrusor underactivity is associated with axonal degeneration, fibrosis, muscle loss, as well as linked neurologic disease, it is not known whether there is an association with the geriatric status $[14,15]$. A study by Benjamin., et al. found that recurrent UTI and markers of obstructive uropathy (calculi, diverticula etc.) may be associated with a worser outcome following prostatectomy because of irreversible changes in the detrusor in this group of patients [16]. Similar findings were resonated in the study by Suhani., et al. where patients with UTI had worse outcomes based on IPSS, Qmax and Qav assessment, and these were replicated in our study as well. In our study, higher PVR was also associated with worse outcomes, which has been reported in other studies also [17]. 
Larger PVR causes chronic bladder distension, poor bladder compliance and hypotonia causing poor detrusor function as well as unsatisfactory post operative recovery following surgery [18]. The study by Suhani., et al. found that prostate size of more than 45 grams on abdominal ultrasound showed statistically significant improvement in their outcome parameters, in comparison to those with smaller size prostate. This finding was noted in our study as well. The study by Hakenberg., et al. also stated that pre operative larger prostate size is related with better symptom improvement after prostatectomy [19]. The strength of our study was that it took a holistic view of the functional outcomes by assessing the symptom improvement (by IPSS) as well as uroflowmetric improvement (Qmax and Qav). The study had a few limitations as well. The study was done in a limited number of patients at a single center on a smaller population group. The evaluation and follow-up were done till 3-months post-TURP. Future studies can collect data over a longer period to take into account the long-term outcomes.

\section{Conclusion}

The results of the study showed that elderly patients (>65years) and patients without UTI before TURP had better functional outcome after TURP. Though larger glands (>45grams) on USG had favourable outcome, grade III glands on DRE were found to have poorer outcome. Poorer outcome was also found in patients with higher grades of bladder trabeculations. However, there was no difference in outcome between catheterised and non-catheterised patients. We conclude that intrinsic factors other than neurological changes in the urinary bladder, which typically occur in long standing urinary obstruction, also significantly affect the results of TURP in patients with BPH. Large multicentric studies are required to extrapolate the results on a larger Indian population.

\section{Bibliography}

1. Arrighi HM., et al. "Natural history of benign prostatic hyperplasia and risk of prostatectomy: the Baltimore longitudinal study of aging". Urology 38.1 (1991): 4-8.

2. Briganti A., et al. "Benign prostatic hyperplasia and its aetiologies”. European Urology 8.13 (2009): 865-871.

3. De Reijke TM and Klarskov P. "Comparative efficacy of two $\alpha 1$-adrenoreceptor antagonists, doxazosin and alfuzosin, in patients with lower urinary tract symptoms from benign prostatic enlargement". BJU International 93.6 (2004): 757-762.
4. Lodh B., et al. "Digital rectal grading of benign prostatic hyperplasia: Where does it stand today?" Journal of Mahatma Gandhi Institute of Medical Sciences 21.1 (2016): 40.

5. Gürbüz C and Drake MJ. "Where can urodynamic testing help assess male lower urinary tract symptoms?" Turkish Journal of Urology 45.3 (2019): 157-163.

6. El-Zawahry A., et al. "The Use of Urodynamics Assessment Before the Surgical Treatment of BPH". Current Urology Report 17.10 (2016): 73.

7. Clement KD., et al. "Invasive urodynamic studies for the management of lower urinary tract symptoms (LUTS) in men with voiding dysfunction". Cochrane Database of Systematic Reviews 28.4 (2015): CD011179.

8. Drake MJ., et al. "Diagnostic Assessment of Lower Urinary Tract Symptoms in Men Considering Prostate Surgery: A Noninferiority Randomised Controlled Trial of Urodynamics in 26 Hospitals". European Urology 78.5 (2020): 701-710.

9. Kaplan SA., et al. "Differential diagnosis of prostatism: a 12year retrospective analysis of symptoms, urodynamics and satisfaction with therapy". Journal of Urology 155 (1996): 1305-1310.

10. Hakenberg OW., et al. "The follow-up of patients with unfavourable early results of transurethral prostatectomy". British Journal of Urology 84 (1999): 799-804.

11. Milonas D., et al. "The effect of complete transurethral resection of the prostate on symptoms, quality of life, and voiding function improvement". Central European Journal of Urology 68 (2015): 169-174.

12. Hakenberg OW., et al. "Preoperative urodynamic and symptom evaluation of patients undergoing transurethral prostatectomy: analysis of variables relevant for outcome". BJU International 91 (2003): 375-379.

13. Hossain MA., et al. "Effects of Transurethral Resection of Prostate (TURP) on Quality of Life in Case of Benign Prostatic Hyperplasia". JNINB 5.2 (2019): 143-147.

14. Aldamanhori R and Chapple CR. "Underactive bladder, detrusor underactivity, definition, symptoms, epidemiology, etiopathogenesis, and risk factors". Current Opinion in Urology 27.3 (2017): 293-299. 
15. Jeong SJ., et al. "Prevalence and clinical features of detrusor underactivity among elderly with lower urinary tract symptoms: a comparison between men and women". Korean Journal of Urology 53.5 (2012): 342-348.

16. Benjamin M., et al. "Factors affecting change in quality of life after prostatectomy: The impact of surgical techniques". Journal of Urology 155 (1996): 191-196.

17. Javle P., et al. "Grading of benign prostatic obstruction can predict the outcome of Transurethral Prostatectomy". Journal of Urology 160.5 (1998): 1713-1717.

18. Jensen KM., et al. "Urodynamics in prostatism. Prognostic values of medium fill water cystometry". Scandinavian Journal of Urology and Nephrology 114 (1988): 78-83.

19. Hakenberg OW., et al. "Does evaluation with the International prostate symptom score predict the outcome of transurethral resection of prostate?" Journal of Urology 158 (1997): 94-99.

Volume 4 Issue 10 October 2021

(c) All rights are reserved by $\mathrm{H}$ Krishna Moorthy., et al. 